\title{
A study on short term outcome of stroke in a rural tertiary care center of Sub Himalayan Terai
}

\author{
Sanchita Saha', Susmita Ghosh², Sisir Chakraborty ${ }^{3}$, Kaushik Ghosh4, \\ Amitava Acharyya ${ }^{5}$, Piyali Kundu ${ }^{6}$, Pasang Lahmu Sherpa ${ }^{7}$, Atanu Biswas ${ }^{8}$ \\ ${ }^{1}$ Assistant Professor, Department of Medicine, IPGMER Hospital, Kolkata, ${ }^{2} R M O$, Department Of Anaesthesiology, \\ Murshidabad Medical College, Berhampore WB, India, ${ }^{3}$ Assistant Professor, Department of Medicine, College of \\ Medicine and Sagore Dutta Hospital, Kolkata, ${ }^{4}$ Assistant Professor, Department of Medicine, Murshidabad Medical \\ College, Berhampore WB, India, ${ }^{5}$ Independent Public Health Consultant, Howrah, ${ }^{6}$ Demonstrator, Department of \\ Pathology, IPGMER Hospital, Kolkata, ${ }^{7}$ Assistant Professor, Department of Medicine, North Bengal Medical College, \\ ${ }^{8}$ Associate Professor, Department of Neurology, IPGMER Hospital, Kolkata
}

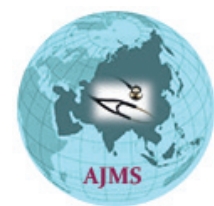

\section{A B S TRACT}

Background: Stroke is the second most common cause of mortality in the world, causing immense morbidity and economic burden. The proper knowledge of the factors which influence the good clinical outcome in stroke is utmost strength of the clinicians in India, where post stroke rehabilitative measures are limited. Aims and Objective: To find out the association of outcome of stroke with different risk factors. Materials and Methods: A descriptive observational design study was formulated for a period of six months on hundred stroke patients in internal medicine unit of at North Bengal Medical College \& Hospital, India. Patients were selected after proper screening by inclusion and exclusion criteria. The "In-hospital-outcome" of CVA patient was determined by Glasgow outcome scale. The neurological condition was determined by National Institute of Health Stroke Scale (NIHSS). The Modified Rankin Scale and Barthel Index were utilized to asses disability status of patient in different period of hospital admission. The data were analyzed by EPI INFO software. Results: Clinical outcome showed statistically significant $(P<0.05)$ correlation with age, sex, level of consciousness, blood pressure, diabetes, past history, mass effect, size and depth of infarction and intra-ventricular extension of intra cerebral bleeding. Other parameters like type of stroke (ischemic or hemorrhagic), delay in admission, dyslipidemia, presence of heart disease, smoking, alcoholism, family history, depth and volume of intra cerebral bleeding, although showed poorer clinical outcome, their correlation was not statistically significant. Conclusion: In-hospital outcome of stroke as determined by Glasgow outcome scale correlates with diverse clinical parameters including various risk factors.

Key words: Dyslipidemia; Glasgow coma scale; Morbidity; North Bengal
http://nepjol.info/index.php/AJMS DOI: 10.3126/ajms.v9i4.19684 E-ISSN: 2091-0576 P-ISSN: 2467-9100

\section{INTRODUCTION}

Stroke is defined (World Health Organization) as "rapidly developing signs of focal or global disturbances of cerebral functions, leading to death or lasting more than 24 hrs with no apparent cause other than vascular origin". ${ }^{1}$ It is a global health problem and is a leading cause of adult disability worldwide. Stroke is the third commonest cause of death after cardiovascular disease and malignancy in India. ${ }^{2}$ A WHO collaborate study showed that both in developed and developing countries, nearly one-third of stroke patients died within 3 weeks and $48 \%$ died within a year. Among all the neurological disease of adult-life, stroke clearly ranks first in frequency and importance, at least half of the neurological disorders in a general hospital are 
of this type. ${ }^{3}$ Studies on stroke epidemiology about incidence, prevalence, and case fatality have mostly been conducted among the developed nation. Indian Council of Medical Research (ICMR) indicates that in 2004 there were 930,985 cases of stroke in India with 639,455 deaths and 6.4 million disability adjusted life years (DALY) lost. ${ }^{4}$ According to the estimates by the National Commission on Macroeconomics and Health, India, there will be 1.67 million stroke cases in India in 2015. ${ }^{5}$ In a recent epidemiological study in the city of Kolkata, the age standardized prevalence rate to world standard population was 545.10 per 100,000 persons. $^{3}$

The patients of stroke either die or live with disabilities. The clinical manifestation depends on the site and size of stroke. Following a stroke, oedema develops in the parenchyma surrounding the damage brain due to inflammatory tissue reaction. This along with increase size of infarct or haemorrhage is responsible for deterioration of patient during early phase. The early mortality following acute stroke is mostly due to swelling of brain leading to cerebral herniation. The other cause of early deterioration following stroke are neurological cause like failure of collaterals blood vessels, recurrent stroke, haemorrhagic transformation of cerebral infraction, cerebral edema, seizure, etc. Systemic factors like - Infection, metabolic disturbances, venous thromboembolism, pulmonary oedema, drugs and cardiovascular complication are also responsible for early mortality. ${ }^{6,7}$ Therefore, initial outcome following an acute stroke depends on successful control of these factors. Despite the advent of recent modalities of treatment of selected patients with acute ischemic stroke, effective prevention remains the best treatment for reducing the disease burden. ${ }^{8-10}$ Primary prevention important because $>70 \%$ of stroke are first ever stroke. ${ }^{11}$ It is helpful for healthcare providers and the public to be able to estimate a person at risk for a first stroke.

However, very little reliable information is currently available regarding epidemiology of stroke in India. The reported frequency, pattern, risk factors, and outcome of stroke from India are largely derived from hospitalbased observations. ${ }^{12,14}$ Very few epidemiological studies on stroke are available from India. ${ }^{3,13,22}$ Hence, in this study, it is planned to determine the subtypes of stroke, evaluate the risk factors of stroke, short term outcome (with available and affordable treatment modalities), case fatality rate, and the evaluation of factors responsible for outcome of stroke in this rural tertiary care center. This study is different from other studies as National Institute of Health Stroke Scale (NIHSS) for assessment of neurological deficits in post stroke patients instead of the Glasgow Coma scale is used.

\section{MATERIALS AND METHODS}

A cross sectional observational study was conducted among the stroke patients admitted in Medicine ward at North Bengal Medical College and Hospital, during June 2010 to May 2011. Participants were selected by purposive sampling procedure. In this study, 167 participants were selected after screening by following inclusion and exclusion criteria. But 17 patients were lost in follow up, so we had followed up 150 patients of acute stroke up to three months.

\section{Inclusion criteria}

1. Clinically suspected cases of stroke patients who were admitted in the General Medicine ward from Neuromedicine or Medicine outpatient and emergency department.

2. Stroke diagnosis confirmed by cranial CT scan.

3. Informed consent from the patients or his/her next of kin (in unconscious patients).

\section{Exclusion criteria}

The patients with following diagnosis were not included in this study. Transient Ischemic Attack, Head Injury, Subarachnoid hemorrhage, Venous stroke (cerebral venous thrombosis), Stroke mimickers (e.g. seizure, intracranial tumors, certain types of migraine, post-ictal phenomenon, hypoglycemia, other metabolic encephalopathy etc.), Acute or chronic meningoencephalitis, Unmasked cases of prior stroke by fever or sepsis, Intracranial hemorrhage within pre-existing brain lesion (e.g. neoplasm).

Each patient was assessed clinically by taking detailed history from the patient or his/her family members, followed by clinical examination at the time of admission and the periodically till discharge or death. The following scales were used for assessment of severity and disability of patients, National Institute of Health Stroke Scale (NIHSS) for acute neurological deficits at the time of admission, then again on $3^{\text {rd }}$ and $7^{\text {th }}$ day and at discharge, Modified Rankin Scale and Barthel Index for disability assessment at $1^{\text {st }}$ and $3^{\text {rd }}$ month of the patient who had survived the ictus. A through clinical examination including general survey and detailed neurological, other systemic examinations were also carried out. All the observations were noted.

Each patient underwent following investigations, cranial CT scan and MRI where indicated (according to inclusion criteria), routine investigations including blood hematology, viz. hemoglobin percentage, ESR, total differential count, blood biochemistry, viz. lipid profile, sugar, creatinine, electrolytes and electrocardiographygraphy. For neuroimaging studies, Computerized Tomography scan single slice spiral, Hitachi CT scan machine of this institution's radiological department were used, and 5,10mm slice were 
taken in most of the cases. In some cases MRI of brain had done using 1.5 Tesla ge MRI machine. Intracranial hematoma volume was calculated according to the formula of ellipsoids $(\mathrm{ABC} / 2){ }^{15}$ The first area of assessment in stroke outcome score is the evaluation of neurological impairment clinically by documenting major domains of involvement. ${ }^{16}$ NIHS Scale was used to assess stroke severity in Motor, Sensory, Vision, Language, Cognition, Affect ${ }^{17}$ during admission, $3^{\text {rd }}$ and $7^{\text {th }}$ day and at discharge. $2^{\text {nd }}$ major area of assessment in determining the stroke outcome is measures of Disability defined as "any restriction or lack of ability to perform an activity in a manner within the range considered normal for a human being." TheBarthel Index and Modified Rankin scale were used for disability assessment. ${ }^{18}$ During analysis we had divided MRS in good outcome with score 0,1,2 and moderate outcome with score 3 , 4. Similarly with barthel index score less than 50 and more than 50 in two groups, though patient is not independent even at the score of 50 .

An approval of the Institutional Ethics Committee and Health University was duly obtained as per existing norms. The written consent was taken from each patients or patients relatives (in unconscious patients).

All the data were collected in a prescribed format.and tabulated in excel sheet (Microsoft office 2007). The data were analyzed by EPI INFO software. The chi square test and $t$ test were performed. $\mathrm{P}$ value $<0.05$ was considered as statistically significant.

\section{RESULTS}

Of these total 150 patients $113(75.33 \%)$ had ICH and $37(24.67 \%)$ had cerebral infraction on neuro-imaging study. Sixty-two patients died during 28 days following stroke and thus the case fatality rate within 28 days was $41.33 \%$. Amongst the 88 survivors after 28 days, 58 were intracerebral hemorrhage and 30 were cerebral infraction. We followed them up till three months of the stroke and found no more death.

Table no. 1 showing that $52 \%$ of total stroke patients were between 41-60 years age group. Fourteen percent of patients were below the age 40 years. It also shows $56.64 \%$ of cases of ICH occurred in 41-65 yrs age group with $30.97 \%$ of male patients. In cerebral infraction male and female $(18.92 \%)$ equally affected. Relation of age and sex of stroke patients (either in ICH or in infraction patients) were statistically insignificant ( $\mathrm{p}$ value $>0.05$ ), denoting age and sex distribution were comparable.

Male, female ratio almost equal in ICH but in cerebral infraction male preponderance $(59.46 \%)$ noted. But calculated $\mathrm{p}$ value $>0.05$ statistically not significant.

In ICH, death rate within one month of post stroke was very high $48.67 \%$ in comparison to Cerebral infraction $(18.92 \%)$. So survival rate of cerebral infraction in onemonth period was high in comparison to intracerebral hemorrhage (95\% CI is 1.53-11.13) which is statistically significant $(\mathrm{p}<0.05)$.

The Table 2 shows that $60 \%$ of patients were admitted within 24 hours of the onset while $40 \%$ came after 24 hours' period. It also shows that only $12 \%$ came within 3 hours of the onset. Fifteen out of 113 patients (13.27\%) with ICH and 3 out of $37(8.10 \%)$ cerebral infraction patients came within 3 hours' periods of the onset. However, despite early arrival, $9(60 \%)$ out of 15 patients of ICH died while one out of three infraction patients $(33.33 \%)$ died during hospital stay. However, there was no difference of early versus late presentation between ICH and Cerebral infraction group $(\mathrm{p}>0.05)$.

This table no. 3 shows the distribution of occurrence of various symptoms at the onset in case of stroke. Among 113 cases of ICH $68.14 \%$ had loss of consciousness (LOC) and among 37 cases of cerebral infraction $29.73 \%$ cases had right sided hemiparesis as most common clinical presentation. On analysis LOC, convulsion, vomiting and speech disorders were found statistically significant in relation to ICH ( $\mathrm{p}$ value $<0.05$ ) where as monoparesis had significant association with cerebral infraction ( $\mathrm{p}$ value $<0.05)$.

This table 4 depicts the distribution of presence of associated risk factors in ICH and cerebral infraction. In cerebral infraction history of RHD was present in $37.84 \%$ of cases

\section{Table 1: Age and Sex distribution of stroke patients observed during study period [ $\mathrm{N}=150]$}

\begin{tabular}{|c|c|c|c|c|c|c|c|}
\hline \multirow[t]{2}{*}{ Age in yrs } & \multicolumn{3}{|c|}{ Intracerebral hemorrhage [n1=113] } & \multicolumn{3}{|c|}{ Cerebral infraction[ $\left.n_{2}=37\right]$} & \multirow[t]{2}{*}{$\mathrm{N}=\mathrm{n}_{1}+\mathrm{n}_{2}$} \\
\hline & Male & Female & Total & Male & Female & Total & \\
\hline $15-40$ & $3(2.65)^{*}$ & $7(6.2)$ & $10(8.85)$ & $7(18.92)$ & $4(10.81)$ & $11(29.73)$ & $21(14)$ \\
\hline $41-60$ & $35(30.97)$ & $29(25.7)$ & $64(56.64)$ & $7(18.92)$ & $7(18.92)$ & $14(37.84)$ & $78(52)$ \\
\hline $61-75$ & $13(11.51)$ & $16(14.12)$ & $29(25.66)$ & 7 (18.92) & $4(10.81)$ & $11(29.73)$ & $40(26.67)$ \\
\hline$\geq 75$ & $5(4.42)$ & $5(4.42)$ & $10(8.85)$ & 0 & $1(2.7)$ & $1(2.7)$ & $11(7.33)$ \\
\hline Total & 56 (49.56) & $57(50.44)$ & 113 & $21(56.76)$ & $16(43.24)$ & 37 & 150 \\
\hline
\end{tabular}


Table 2: Postictal delay in arriving at institution among study population [N=150]

\begin{tabular}{|c|c|c|c|c|c|c|c|}
\hline \multirow[t]{2}{*}{ Duration } & \multicolumn{3}{|c|}{ Intracerebral hemorrhage $\left(n_{1}=113\right)$} & \multicolumn{3}{|c|}{ Cerebral infraction $\left(n_{2}=37\right)$} & \multirow{2}{*}{$\begin{array}{c}\text { Total } \\
\mathrm{N}=\mathrm{n}_{1}+\mathrm{n}_{2}\end{array}$} \\
\hline & Alive & Died & Total & Alive & Died & Total & \\
\hline$<3 \mathrm{hrs}$ & $6(4)^{*}$ & $9(6)$ & $15(13.27)$ & $2(1.3)$ & $1(0.7)$ & $3(8.10)$ & $18(12)$ \\
\hline $3-24$ & $29(19.4)$ & $26(17.3)$ & 55 (48.67) & $15(10)$ & $2(1.3)$ & 17 (45.95) & $72(48)$ \\
\hline$>24$ hrs. & $23(15.3)$ & $20(13.4)$ & $43(38.05)$ & $13(8.7)$ & $4(2.6)$ & 17 (45.95) & $60(40)$ \\
\hline Total & $58(38.7)$ & 55 (36.7) & 113 & $30(20)$ & $7(4.6)$ & 37 & $150(100)$ \\
\hline
\end{tabular}

Table 3: Clinical features observed among admitted stroke patient during study period

\begin{tabular}{lccccc} 
Clinical features & Cerebral infraction $\mathbf{~ N}_{\mathbf{1}} \mathbf{= 3 7}$ & $\mathbf{I C H} \mathbf{~ N}_{\mathbf{2}}=\mathbf{1 1 3}$ & Total & $\mathbf{p}$ value & $\mathbf{9 5 \%} \mathbf{C I}$ \\
\hline Loss of consciousness & $9(24.32)^{*}$ & $77(68.14)$ & 86 & 0.0000 & $0.06-0.38$ \\
Altered sensorium & $3(8.11)$ & $8(7.08)$ & 11 & 0.8350 & $0.23-5.2$ \\
Convulsion & $7(18.92)$ & $45(39.82)$ & 52 & 0.0203 & $0.13-0.93$ \\
Vomiting & $9(24.32)$ & $60(53.1)$ & 69 & 0.0023 & $0.11-0.70$ \\
Speech disorder & $13(11.51)$ & $21(18.58)$ & 34 & 0.0368 & $0.96-5.85$ \\
Rt hemiparesis & $11(29.73)$ & $29(25.64)$ & 40 & 0.6273 & $0.5-2.99$ \\
Lt hemiparesis & $11(29.73)$ & $19(16.82)$ & 30 & 0.0835 & $0.81-5.36$ \\
Monoparesis & $3(8.11)$ & $1(0.89)$ & 4 & 0.0179 & $0.87-255.12$ \\
$7^{\text {th }}$ cranial palsy UMN & $29(78.38)$ & $89(78.76)$ & 118 & 0.9606 & $0.35-2.58$ \\
Dizziness & $6(16.21)$ & $10(8.85)$ & 16 & 0.2076 & $0.59-6.61$ \\
Headache & $7(18.92)$ & $25(22.12)$ & 32 & 0.6795 & $0.29-2.26$ \\
\hline
\end{tabular}

\begin{tabular}{|c|c|c|c|c|c|c|}
\hline Serial no. & Risk factors & Cerebral Infraction $\left[n_{1}=37\right]$ & $\mathrm{ICH}\left[\mathrm{n}_{2}=113\right]$ & Total $[n=150]$ & $p$ value & $95 \% \mathrm{Cl}$ \\
\hline 1 & Hypertension & $10(27.01)^{\star}$ & $64(56.64)$ & 74 & 0.0017 & $0.12-0.68$ \\
\hline 2 & Smoking & $7(18.92)$ & $62(54.87)$ & 69 & 0.0001 & $0.13-0.51$ \\
\hline 3 & Diabetes & $8(21.62)$ & $6(5.31)$ & 14 & 0.0030 & $1.40-17.65$ \\
\hline 4 & TIA & $1(2.71)$ & $1(0.89)$ & 2 & 0.4027 & $0.0-117.33$ \\
\hline 5 & Recurrent stroke & $2(5.41)$ & $13(11.51)$ & 15 & 0.2831 & $0.06-2.21$ \\
\hline 6 & RHD & $14(37.84)$ & 0 & & 0.0000 & $4.07-8.58$ \\
\hline 7 & $\mathrm{AF}$ & $14(37.84)$ & $3(2.65)$ & 17 & 0.0000 & $5.36-107.35$ \\
\hline 8 & IHD & $5(13.51)$ & 14 (12.39) & 19 & 0.8583 & $0.32-3.64$ \\
\hline 9 & Carotid bruit & $2(5.41)$ & $3(2.65)$ & 5 & 0.4185 & $0.23-16.30$ \\
\hline 10 & Alcohol intake & $2(5.41)$ & 15 (13.27) & 17 & 0.1900 & $0.06-1.84$ \\
\hline
\end{tabular}

\section{Table 5: Distribution of study population according to involvement of cerebral arteries [ $\mathrm{N}=150]$}

\begin{tabular}{lccc} 
Artery involved & Cerebral infraction $\left[\mathrm{n}_{\mathbf{1}}\right]$ & Cerebral hemorrhage $\left[\mathrm{n}_{\mathbf{2}}\right]$ & Total $\left[\mathbf{N}=\mathrm{n}_{\mathbf{1}}+\mathrm{n}_{\mathbf{2}}\right]$ \\
\hline ACA & 0 & 0 & 0 \\
MCA & $24(64.86)^{*}$ & $99(87.61)$ & $123(82)$ \\
PCA & $3(8.10)$ & $10(8.85)$ & $13(8.67)$ \\
Vertebro-basilar & 0 & 0 & 0 \\
Primary IVH & 0 & $2(1.77)$ & $2(1.33)$ \\
Multiple arterial territory & $10(27.03)$ & $2(1.77)$ & $12(8)$ \\
Total & $37(100)$ & $113(100)$ & 150
\end{tabular}

whereas no patients of ICH had similar history. History of hypertension was present in $56.64 \%$ of cases of ICH though most of them are on irregular medications. Significant association ( $\mathrm{p}$ value $<0.05$ ) were found with hypertension ( $95 \%$ CI 0.12-0.6) and with smoking (95\% CI, 0.13-0.51) in ICH and in cerebral infraction with diabetes $(95 \% \mathrm{CI}, 1.40-17.65)$.

Among all the stroke patients $82 \%$ cases had MCA territory involvement, followed by PCA ( $8.67 \%)$ and multiple arterial territories (8\%). Among study population, multiple arterial territories involvement was more common in cases of cerebral infarction $(27.03 \%)$ than in ICH patients $(1.77 \%)$ (Table 5)

Most common site of intracranial haemorrhage was found to be basal ganglia and thalamus (64.6\%) followed by lobar haemorrhage $(23 \%)$. Two cases of primary intraventricular haemorrhage were detected (Table 6).

Most common site of cerebral infraction was subcortical $(56.76 \%)$ followed by involvement of multiple area (Table 7). 
Table 8 shows stage II hypertension was most commonly seen in patients with intracerebral haemorrhage $74.34 \%$ where as in cerebral infraction $27.03 \%$ cases was in pre hypertension stage and in stage II. Significant association was found with stage II hypertension and intracerebral haemorrhage ( $\mathrm{p}$ value $<0.05)$.

Seventy eight point seventy six percent cases of intracerebral haemorrhage had stage II hypertension according to JNC7 where as $21.62 \%$ in cases of cerebral infraction were in stage II hypertension. On statistical analysis significant association was found with ICH stage II hypertension ( $\mathrm{p}$ value $<0.05)$.

Most common age of presentation of intracerebral haemorrhage was more than $40 \mathrm{yrs}$. And complete recovery occurred in 5 cases $(4.43 \%)$ with age less than 40 yrs. On statistical analysis significant association was found with outcome of stroke in relation to younger age of patient ( $\mathrm{p}$ value $<0.05)$ (Figure 1).

Most common age of presentation of cerebral infraction was $>40$ years of age. Death and residual paralysis was more in $>40$ years age group compared to younger. Complete recovery was more seen in $<40$ years age group. These association were statistically significant ( $\mathrm{p}$ value $<0.05$ ) (Figure 2).

Intracranial hematoma volume $>30 \mathrm{cc}$, intraventricular extension of intracerebral hemorrhage and midline shift $>5 \mathrm{~mm}$ are well predictors of poor outcome of intracerebral hemorrhage. On statistical analysis all were found to be significant ( $p$ value $<0.05$ ).

Total 13 cases $(11.5 \%)$ had recurrent stroke, rest 100 cases had first ever stroke among ICH stroke patients. On

\begin{tabular}{lcc} 
Table 6: Site of Haemorrhage among stroke \\
patients with ICH observed during study period \\
[N=113] \\
Area of bleed & No of cases & \% of Total \\
\hline Basal ganglia, thalamus & 73 & 64.60 \\
Lobar hemorrhage & 26 & 23.01 \\
Cerebellar & 6 & 5.31 \\
Brainstem & 6 & 5.31 \\
Primary IVH & 2 & 1.77 \\
Total & 113 & 100 \\
\hline
\end{tabular}

Table 7: Area of infarction among stroke patients

\begin{tabular}{lcc} 
Area of bleed & No of cases & \% of total \\
\hline Subcortical & 21 & 56.76 \\
Cortical & 5 & 13.51 \\
Cerebellar & 1 & 2.71 \\
Brainstem & 2 & 5.4 \\
Multiple area & 8 & 21.62 \\
Total & 37 & 100 \\
\hline
\end{tabular}

Asian Journal of Medical Sciences | Jul-Aug 2018 | Vol 9 | Issue 4 analysis statistical significance were not found in relation to death ( $\mathrm{p}$ value $>0.05)$.

Seventy five point eight six percent of $\mathrm{ICH}$ patients who were admitted with severe neurological deficits (by NIHSS score) were improved to $31.03 \%$ cases with moderate neurological deficits at the end of third month of follow up.

In cerebral infractions $53.33 \%$ patients were admitted with severe neurological deficits but at the end of $1^{\text {st }}$ month of follow up only $3.33 \%$ remain severely neurodeficit and at the end of $3^{\text {rd }}$ month only $20 \%$ cases remain moderately neurodeficit with 6-15 NIHSS score.

Seventy five point eight six percent of ICH cases who were admitted with severe neurological deficits with NIHSS score of 16 to 42 was improved to $34.5 \%$ at the end of $3^{\text {rd }}$ month of follow up in comparison to $63.8 \%$ at the end of $1^{\text {st }}$ month.

In cerebral infraction $53.33 \%$ cases were admitted with NIHSS score of 16 to 42 and at the end of $3^{\text {rd }}$ month of follow up only $26.66 \%$ of patients remains as moderately disabled with Modified Rankin score of either 3 or 4 .

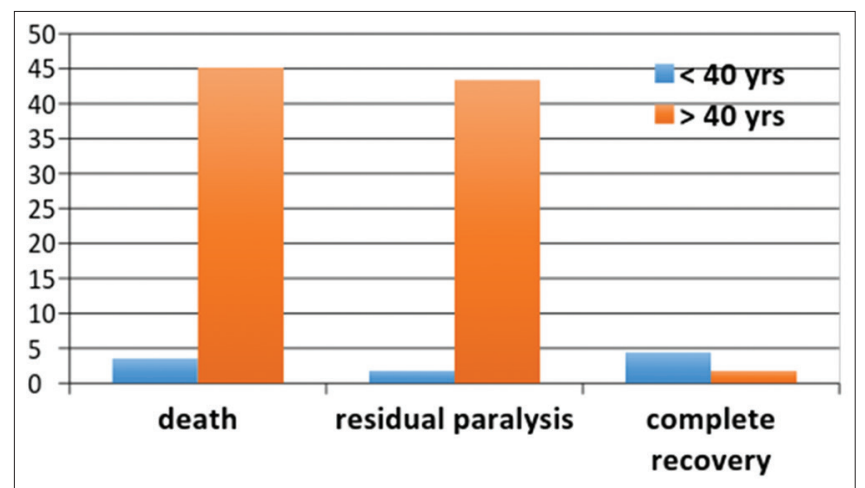

Figure 1: comparison between outcomes of $\mathrm{ICH}$ at $1^{\text {st }}$ month with age group among study population

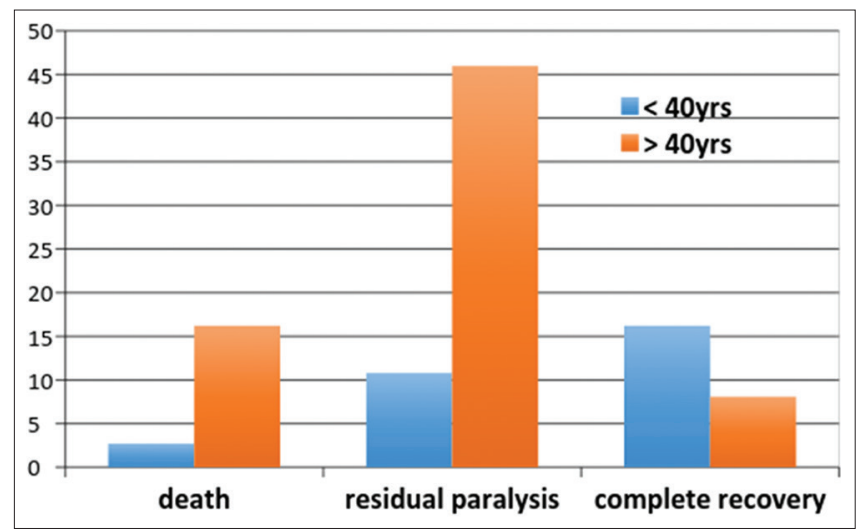

Figure 2: Comparison between outcomes of cerebral infraction at $1^{\text {st }}$ month in relation to age-groups among study population 
Table 8: Systolic BP at admission of ICH and Cerebral Infarction patients [N=150]

\begin{tabular}{lccc} 
SBP $(\mathbf{m m}$ of $\mathbf{H g})$ & Intracranial hemorrhage & Cerebral infraction & Total \\
\hline Normal $(<120)$ & $5(4.24)^{\star}$ & $7(18.92)$ & $12(8)$ \\
Pre Hypertension ( $120-139)$ & $5(4.24)$ & $10(27.03)$ & $15(10)$ \\
Stage I Hypertension $(140-159)$ & $19(16.82)$ & $10(27.03)$ & $29(19.33)$ \\
Stage II Hypertension (160 or more ) & $84(74.34)$ & $10(27.03)$ & $94(62.67)$ \\
\hline
\end{tabular}

Barthel Index is also a tool of disability assessment. Those 44 patients $(75.86 \%)$ of ICH who were presented with severe neurological deficits improved with remaining 10 patients $(17.24 \%)$ as severely disabled and dependended with barthel score $\leq 50$ at the end of three month of follow up.

In cases of cerebral infraction only 2 patients $(6.67 \%)$ remained severely disabled and depended at the end of study period.

Figure 4 and 5 shows associations of risk factors with $\mathrm{ICH}$ and cerebral infraction in relation to outcome in the form of 30-days mortality, partial recovery and complete recovery were assessed. Significant association was found with outcomes in relation to age and hypertension in cases of cerebral infraction and in cases of $\mathrm{ICH}$ to age and smoking ( $p$ value $<0.05$ ). It failed to show any statistical significance with other risk factors in relation to different outcomes may be due small sample size.

\section{DISCUSSION}

Acute stroke is a heterogeneous condition with respect to prognosis. It is impossible to predict outcome in an individual with greater accuracy. In stroke chances of survival depend on various factors like neurological damage, metabolic disturbance and systemic dysfunction. Early mortality rate in this study was $41.33 \%$ (95\% CI, 1.54 to 11.13 ) which is much higher that observed in developed nations $(17 \%$ to $33 \%) .{ }^{13}$ In this study one-month mortality from stroke or related complications in ICH is $48.67 \%$ and in cerebral infraction $18.92 \%$. Daverat P et al.shows $31 \%$, Douglas and Haerer reported a mortality rate of $40 \%{ }^{19,20}$ In Oxfordshire ${ }^{21}$ case fatality rates $10(95 \% \mathrm{CI}, 7$ to 13$)$ and $50(95 \%$, CI 38 to 62) for cerebral infraction and ICH respectively and in Perth study it was 12 (95\% CI, 8 to 16$)$ and 30 (95\%, CI 17 to 44). In a population based stroke survey in Mumbai, India Dalal et al.shows overall case fatality for first ever stroke was $29.8 \%{ }^{22}$ The higher 30-day case fatality ratio in our study is probably due to inclusion of more severe form of the cases. This is because it is a hospital based study from a tertiary referral centre. Minor strokes or those with intact consciousness are usually managed at home or in the primary care centres and they are often not referred or brought to the hospital.

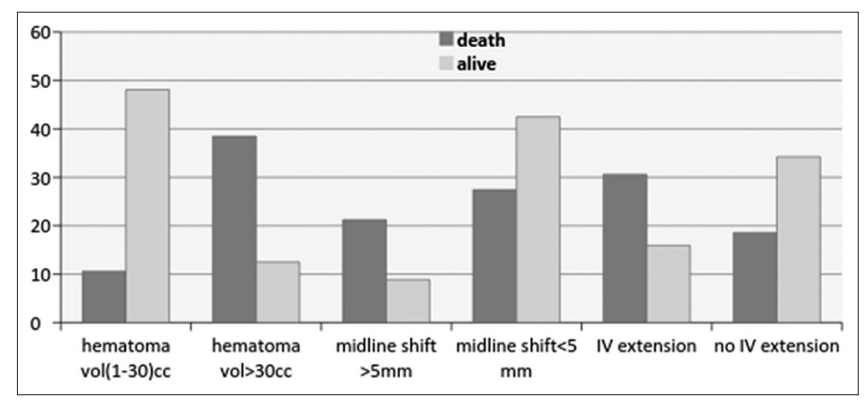

Figure 3: Association of important CT scan finding with outcome in intracerebral hemorrhage

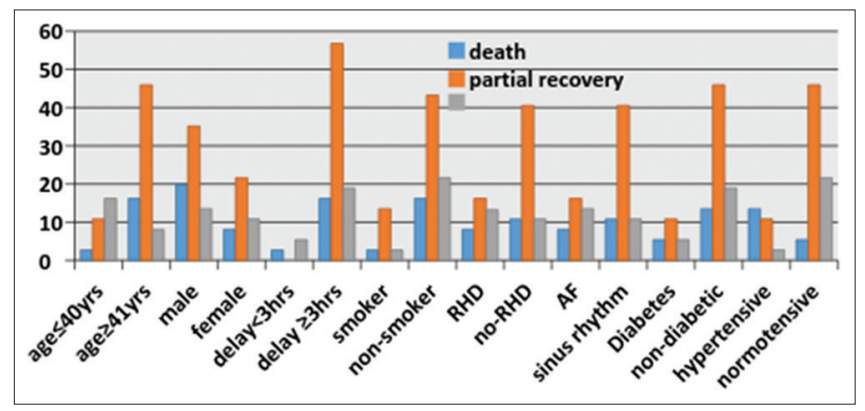

Figure 4: Association of risk factors with outcome of cerebral infraction at $1^{\text {st }}$ month among study population

In this study there were $75.33 \%$ cases of intracerebralhemorrhage and $24.64 \%$ cases of cerebral infraction. The ratio between cerebral infraction: hemorrhage was 0.33 excluding subarachnoid hemorrhage. According to Feigin et al.2009 ischemic stroke accounts for 50\% - 85\% and hemorrhagic stroke either subarachnoid or ICH accounts for $(1 \%-7 \%)$ or $(7 \%-27 \%)$ respectively worldwide. ${ }^{23}$ In Kolkata study, 11 neuro-imaging was performed soon after stroke in $59.5 \%$ cases which revealed cerebral infraction in $68 \%$ and ICH $32 \%$ cases. The ratio between cerebral infraction and $\mathrm{ICH}$ was 2.21 where as in our study the ratio was 0.33 . This maximum cases of $\mathrm{ICH}$ can be explained as our institution is a referral tertiary care centre situated in a rural area and apex referral centre of a large northern part of West Bengal with a very poor socio-economic and cultural as well educational background with unhealthy life style. So the less severe strokes either not referred from primary health centre or patient neglect due to poverty or lack of awareness or managed by local physician etc.

Sixty percent of our patients were admitted within 24 hours of the onset while $40 \%$ (45.94\% of cerebral infraction 


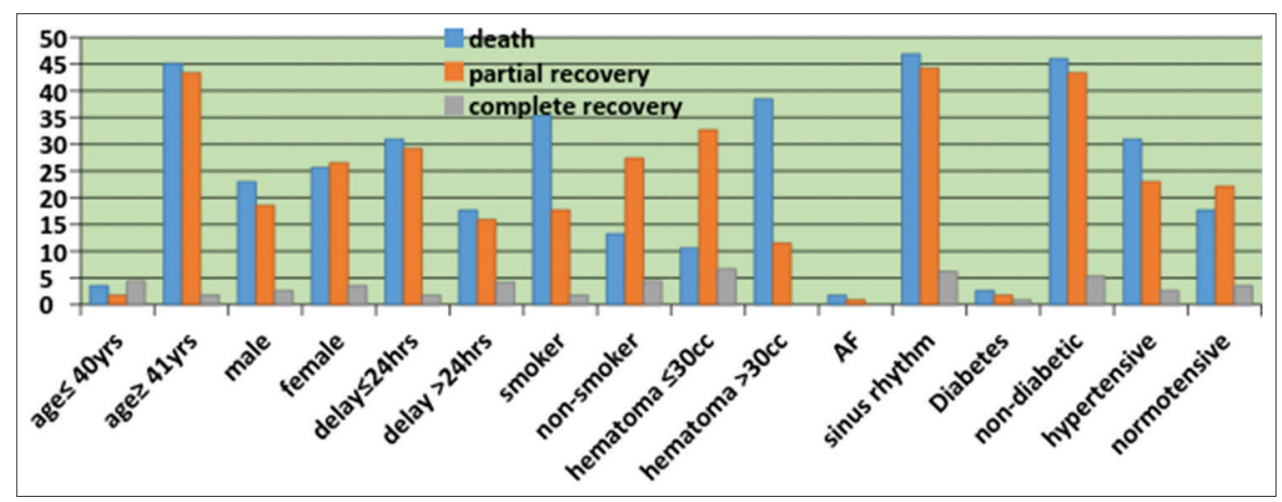

Figure 5: association of risk factors with outcome of $\mathrm{ICH}$ at $1^{\text {st }}$ month among study population

and $38.05 \%$ of ICH) came after 24 hours' period. Only $12 \%$ came within 3 hours of the onset. Fifteen out of 113 patients $(13.27 \%)$ with ICH and 3 out of thirty-seven $(8.10 \%)$ with cerebral infraction came within 3 hours' period. However, despite early arrival, 9 put of $15 \mathrm{ICH}$ patients $(60 \%)$ died while 1 out of 3 infraction patients $(33.33 \%)$ died during hospital stay. However, there was no difference of early versus late presentation between ICH and infraction group ( $\mathrm{p}>0.05)$. Nagaraja and colleagues in Bangalore, India had shown delayed arrival of stroke patients (44\% reached hospital after 48 hrs of stroke occurrence) ${ }^{22}$ which is consistent with our study $45.94 \%$ patients of cerebral infraction reached hospital after $24 \mathrm{hrs}$ of stroke, which precludes the scope of modern treatment $(7.96 \%)$ patients of ICH were admitted within 3 hours of stroke but outcome was death may be explained by severity of neurological insult. $48.66 \%$ of ICH patients were admitted within 24 hours of stroke.

Das et al. ${ }^{3}$ in a study in Kolkata had shown the ratio of cerebral infraction: ICH was 1.86. In ICH basal-ganglia thalamic region was the most common $(75 \%)$ site of intracranial bleed, followed by lobar (12.5\%), cerebellar $(5 \%)$, brain stem $(5 \%)$, primary intraventricular region $(2.5 \%)$. But in our study higher incidence of lobar hemorrhage (23.01\%) detected [vide Table-8]. In our study in cerebral infraction, the most common lesions were subcortical $(56.76 \%)$, followed by multiple area $(21.62 \%)$, cortex (13.51\%) brainstem (2.71\%) and cerebellum (5.4\%) whereas Das et al. ${ }^{3}$ had shown in their study most common lesion were subcortical (75.6\%),102 followed by cortex $(19.8 \%)$, brainstem $(2.3 \%)$, and in cerebellum $(2.3 \%)$.

In this study $11.51 \%$ of ICH and $5.41 \%$ of cerebral infraction patients had prior history of stroke (recurrent stroke). $2.71 \%$ and $0.89 \%$ of infraction and $\mathrm{ICH}$ respectively had given history of TIA.

Associated Risk Factors: The Framingham Heart Study and other international prospective epidemiological studies identified the major atherogenic risk factors for stroke as hypertension, diabetes mellitus, hyperlipidemia, and smoking. ${ }^{24}$

Hypertension: In our study 56.64\% cases of ICH were known hypertensive but on irregular medications and $27.01 \%$ of cerebral infraction cases were hypertensive (JNC 7 criteria) with significant association ( $\mathrm{p}$ value $<0.01$ and $95 \%$ CI 0.12 0.6). A community based case-control study ${ }^{13}$ in Kolkata had demonstrated hypertension was the most important risk factor stroke with odds ratio of 5.04 (95\% CI 4.16-5.92) in women and 21.87 (95\% CI 18.69-25.05) in men. ${ }^{7}$

Diabetes Mellitus: In the present study $21.62 \%$ cases of infraction and only $5.31 \%$ cases of ICH were diabetic. Significant association was found with cerebral infraction $(\mathrm{p}<0.01$ and $95 \%$ CI, 1.40-17.65). However, DM is known to hasten atherosclerosis which in turn favours the development of cerebral infraction.

Ischemic Heart Disease: In our study only $13.51 \%$ of cerebral infraction and $12.39 \%$ of ICH had IHD and calculated $\mathrm{p}$ value $>0.05$. Emboli tend to occur in the first few weeks after an acute myocardial infarction but one study had found that a lesser degree of risk persist for up to 5 yrs. ${ }^{3}$

Rheumatic Heart Disease: In this study $37.84 \%$ of cerebral infraction cases associated with valvular heart diseases, but it failed to show any statistical significance in respect to outcome ( $p$ value $>0.05$ ). But the presence of atrial fibrillation in $37.84 \%$ case of cerebral infraction was statistically significant, as calculated $\mathrm{p}$ value $<0.01$.

Smoking: In this study $18.92 \%$ of infraction and $54.87 \%$ of ICH cases were smoker and it was statistically significant ( $\mathrm{p}$ value $<0.05$ and $95 \%$ CI 0.13-0.51).

Clinical features: This study found most common clinical presentation of hemorrhagic stroke was initial transient 
loss of consciousness in 77 cases $(68.14 \%)$ with 95\% CI of $0.06-0.38$ followed by vomiting and convulsion. In ischemic stroke the most common presentation right sided hemiparesis, followed by transient loss of consciousness, and vomiting.

The NIH Stroke Scale is a convenient, rapidly applied scale for neurological assessment that correlates with other measures of stroke such as infract size on CT scan. Because of its simplicity and high interrater reliability, it is well suited for clinical assessment trial of acute stroke, in which serial assessment may be performed by different persons. Since in this study assessments were performed by one individual, variability in application of stroke score should have been small. Wityk et al. ${ }^{5}$ found admission NIHSS score as an important predictor of outcome. They found early change from admission to $24 \mathrm{hrs}$ are important predictors at 7 to 10 days and follow up. In this study $29.31 \%$ cases of ICH at the end of $1^{\text {st }}$ month and none at the end of $3^{\text {rd }}$ month remain severely disabled with NIHSS score of, $73.33 \%$ of patients of cerebral infraction was severely disabled at time of admission but at the end of study period all improved. Nearly $63.8 \%$ cases of ICH and $50 \%$ of infraction were significantly disabled (Rankin score $\geq 3$ ) 1 month post stroke, which is very similar to the Trivandrum Stroke Registry ${ }^{6}$ where they found $60 \%$. It is also very similar to the outcome of patients from the Polish National Stroke Registry. ${ }^{7}$ Our inability to find more disability among older patients may be related to relatively small number of very old patients.

\section{Limitations of the study}

The important limitations of our study are

1. The main limitation of this study was that the study population was small. Therefore, the results of this study did not simulate many other epidemiological studies conducted elsewhere.

2. It is a prospective hospital based non-interventional observational study. So the actual scenario of stroke remains undetected. Only those patients where stroke is moderate to severe attended Outpatient Department or Emergency of our institution or other hospitals. Mild cases remain undetected. Only tip of the iceberg seen.

3. The other chief shortcoming of the study is referral bias.

4. Since this is a hospital based study, the results were not a true reflection of the disease incidence and prevalence in the community.

\section{REFERENCES}

1. WHO MONICA Project Investigators. The World Health Organization MONICA Project (Monitoring trends and determinants in cardiovascular disease). J Clin Epidemiol 1988; 41: 105-114.

2. Dalal $P$, Bhattacharjee $M$, Vairala $J$ and Bhat $P$. UN millennium development goals: can we halt the stroke epidemic in India? Ann Indian Acad Neural 2007; 10:130-136.

3. Banerjee TK, Mukherjee CS and Sarkhel A. Stroke in urban population of Calcutta: an epidemiological study. Neuroepidemiology 2001;20:201-207.

4. Prasad K and Singhal KK. Stroke in young: An Indian perspective. Neurology India 2010; 58: 343-350.

5. American Heart Association. Heart Disease and Stroke Statistics-2004 Update. Dallas, Tex: American Heart Association; 2003.

6. Workshop report on stroke surveillance in India. WHO. New Delhi. Available from: http://www.whoindia.org/./NMH_ Resources_cvd_MGMT_icmrSTROKE_SURVEILLANCE.pdf

7. Adams H, Adams R, Del Zoppo G and Goldstein LB. Stroke Council of the American Heart Association; American Stroke Association. Guidelines for the early management of patients with ischemic stroke: 2005 guidelines update: a scientific statement from the Stroke Council of the American Heart Association/American Stroke Association (published corrections appear in Stroke 2005;36:1626 and Stroke 2005;36:1352). Stroke 2005;36:916-923.

8. Rundek T and Sacco RL. Risk Factor Management to Prevent First Stroke. Neurol Clin 2008; 26(4): 1007-ix.

9. Green DM, Ropper AH, Kronmal RA, Psaty BM and Burke GL. Serum potassium level and dietary intake a risk factor for stroke. Neurology 2002;59:314-320.

10. Chobanian AV, Bakris GL, Black HR, Cushman WC, Green LA, Izzo JL, et al. National Heart, Lung, and Blood Institute Joint National committee on Prevention, Detection, Evaluation, and Treatment of High Blood Pressure: the JNC 7 report [published correction appears in JAMA.2003;290:197].JAMA 2003;289:2560-2572.

11. American Heart Association. Heart disease and stroke statistics-2004 update. American heart association; Dallas (TX): 2003.

12. Galen (131-200 AD). Coted by Clerk E and Dewhursk K in "An illustrated history of brain function" Los Angles. 1881;1:72.

13. Das Shyamal K, Banarjee TK, Biswas A, Roy T, Raut DK, Mukherjee CS, et al. A prospective community-based study of stroke in Kolkata, India. Stroke 2007; 38:906-910.

14. Govt. of India, Central Bureau of Health Intelligence, National Health Profile 2008. New Delhi: Directorate General of Health Science 2008; p.102.

15. Kothari RU, Brott $T$, Broderick JP, Barsan WG, Sauerbeck LR, Zuccarello M, et al. The ABCs of measuring intracerebral hemorrhage volumes. Stroke 1996; 27:1304- 1305.

16. Manhoney FI and Barthel D. "Functional evaluation the Barthel Index". Maryland State Medical Journal 1965; 14:56-61.

17. National Institute of Health, National Institute of Neurological Disorders and Stroke. Stroke Scale. http://www.ninds.nih.gov/ doctors/NIH_Stroke_Scale.pdf

18. Naik BK, Rao PS and Saboo R. Ind. Heart J. 1966; 18:37.

19. Daverat $P$, Castel JP, Dartigues JF and Orgogozo JM. Death and functional outcome after spontaneous intracerebral hemorrhage. A prospective study of 166 cases using multivariate analysis. Stroke 1991;22:1-6.

20. Douglas MA and Haerer AF. Long-term prognosis of hypertensive intracerebral hemorrhage. Stroke 1985; 12:267-271.

21. Anderson CS, Jamrozik KD, Burvil PW, Chakera TMH, Johnson GA and Stewart-Wynne EG. Determining the incidence of different subtypes of stroke: results from the Perth Community 
Stroke Study, 1989-1990. Med J Aust 1993;158:85-89.

22. Nagaraja D, Gururaj G, Girish N, Panda S, Roy AK, Sharma GRK, et al. feasibility study of stroke surveillance: Data from Bangalore, India. Indian J Med Res 2009; 130:396-403.

23. Asia Pacific Cohort Studies Collaboration. Cholesterol, coronary heart disease, and stroke in the Asia Pacific region. International Journal of Epidemiology 2003; 32:563-572.
24. Diringer MN, Edwards DF and Zaulia AR. Hydrocephalus: A previously unrecognized predictors of poor outcome from supratentorial intracerebral hemorrhage. Stroke 1998; 29:1352.

25. Wityk RJ, Pessin MS and Caplan LR. Serial assessment of acute stroke using the NIH Stroke Scale [published erratum appears in Stroke 1994; 25(6):1300]. Stroke 1994; 25:362-365.

Authors Contribution:

SS,KG, AB- Concept and design of the study, manuscript preparation, critical revision of the manuscript, statistically analyzed and interpreted; SG, SC, PK,

PLS- Concept and design of the study, helped in preparing first draft of manuscript, and review of the study; AA-Study design, reviewed the literature, critical revision of manuscript, statistically analyzed and interpreted.

\section{Orcid ID:}

Dr. Kaushik Ghosh- (1) http://orcid.org/0000-0002-2972-6441

Dr. Sisir Chakraborty- (1) http://orcid.org/0000-0002-8483-9406

Mr. Amitava Acharyya- (1) http://orcid.org/0000-0002-7839-9563

Source of Support: Nil, Conflict of Interest: None declared. 